\title{
Characterization of the Relationship of CDKL5 with MeCP2 and Dnmt1 in Primary Rat Cortical Neurons
}

\author{
Zhi, Yi ${ }^{1}$; Wenxu, Yang ${ }^{1}$; Yinan, Ma' ${ }^{1}$ Yu, Qi1; Hong, Pan ${ }^{1}{ }^{*}$ \\ ${ }^{1}$ Department of Central Laboratory, Peking University First Hospital, NO.8 Xishiku Street, Xicheng District, Beijing \\ 100034, China.
}

\begin{abstract}
Cyclin-dependent kinase-like 5 (CDKL5) is a protein kinase that is homologous to mitogen-activated protein kinases $(M A P K s)$ and cyclin-dependent kinases (CDKs). Mutations in the CDKL5 gene cause X-linked infantile spasms and phenotypes that overlap with that of Rett syndrome, which is a neurodevelopmental disorder caused primarily by mutations in the methyl CpG binding protein 2 gene (MECP2). Previous studies in transfected cell lines showed that CDKL5 interacts with MeCP2 and DNA (cytosine-5)-methyltransferase 1 (Dnmt1). However, little is known about the relationships of CDKL5 with interacting proteins in primary neuronal cultures. In this study, we investigated the expression patterns of CDKL5, MeCP2 and Dnmt1, and their interaction in cultured rat cortical neurons. Using real-time PCR analysis, we found that CDKL5, MeCP2 and Dnmt1 have similar expression patterns at the mRNA level. In contrast, the expression patterns of those proteins at the protein level are different and could be inversely correlated, as shown by western blotting. Using co-immunoprecipitation, we further demonstrated that CDKL5 interacts with MeCP2 and Dnmt1 in primary rat cortical neurons. These data suggest that a functional link exists among CDKL5, MeCP2 and Dnmt1 during neuronal development and may provide further insight into the pathogenesis of Rett syndrome.
\end{abstract}

Key words: CDKL5; MeCP2; Dnmt1; neurodevelopment; primary neurons

\footnotetext{
*Corresponding author: address: panmuren@263.net
} 


\section{INTRODUCTION}

Cyclin-dependent kinase-like 5 (CDKL5), which is also known as serine/threonine kinase 9 (STK9), is a protein kinase that is widely distributed in all tissues and highly expressed in the brain (Lin et al. 2005). CDKL5 is homologous to mitogenactivated protein kinases (MAPKs) and cyclindependent kinases (CDKs). Mutations in the gene that encodes CDKL5 cause intellectual disability, infantile spasms, and variant form of Rett syndrome, which is a neurodevelopmental disorder that is caused primarily by mutations in the methyl $\mathrm{CpG}$ binding protein 2 gene $(M E C P 2)$ (Evans et al. 2005; Kalscheuer et al. 2003; Mari et al. 2005; Tao et al. 2004; Weaving et al. 2004). Because mutations in CDKL5 and MECP2 can cause similar phenotypes in patients, it is possible that CDKL5 and MeCP2 share the same molecular pathway in the central nervous system. Investigating the relationship of CDKL5 with $\mathrm{MeCP} 2$ and other interactors will help to further elucidate the critical roles of CDKL5 and MeCP2 in neural development, plasticity and neurological disorders.

A small number of studies have been performed in vitro or in transfected cell lines to characterize the associations among CDKL5, MeCP2 and other molecules. These studies suggested that CDKL5 and MeCP2 interact (Mari et al. 2005). In addition, CDKL5 was found to bind and phosphorylate DNA (cytosine-5)methyltransferase 1 (Dnmt1), which also associates with MeCP2 (Kameshita et al. 2008; Kimura and Shiota 2003). However, few studies have been carried out in neuronal systems to evaluate the connections among CDKL5, MeCP2 and other interacting proteins.

In this study, we focused on the CDKL5 expression profile and its relationship with $\mathrm{MeCP} 2$ and Dnmt1 during the development of cultured rat cortical neurons, aiming to elucidate the role of CDKL5 and its interactors in the neuronal development process.

\section{MATERIALS AND METHODS}

\section{Rat embryonic cortical neuron cultures}

This study was approved by the Experimental Animal Ethics Committee of Peking University First Hospital (protocol number J201223). Primary cortical neurons were prepared from the brains of embryonic day 18 (E18) Sprague Dawley (SD) rats. Cell culture was performed as described previously (Zhang et al. 2006), with the following modifications. Cortical tissue from fetal rats was carefully dissected and digested with $0.25 \%$ Trypsin (Gibco) at $37^{\circ} \mathrm{C}$ under $5 \% \mathrm{CO}_{2}$ for 5-8 $\mathrm{min}$ in $3.5 \mathrm{~cm}$ dishes. The digestion was terminated by the addition of 6-8 $\mathrm{ml}$ of Dulbecco Modified Eagle Medium (DMEM) (Gibco) supplemented with $10 \%$ FBS (Gibco). Then, the tissue was scattered with pipettes. The separated neurons were plated on poly-L-lysine-coated (Sigma) dishes and maintained first in DMEM with 10\% FBS for 2-4 hours and then in Neurobasal ${ }^{\circledR}$ Medium (Gibco) supplemented with $2 \%$ B-27 Supplement (Gibco) and 1\% LGlutamine (200 mM, Gibco). Every other day, $50 \%$ of the medium volume was replaced.

\section{Immunofluorescence}

The purity of the cultured neurons was determined using immunofluorescence and confocal laser scanning microscopy (Olympus FV1000). Cortical neurons were plated on poly-L-lysinecoated slides (Sigma) at a density of $3 \times 10^{5}$ cells per slide $(24 \mathrm{~mm} \times 24 \mathrm{~mm}$ ). Cells at DIV (days in vitro) $1,4,7,11$ and 15 were fixed with freshly prepared $4 \%$ paraformaldehyde in phosphatebuffered saline (PBS). Non-specific binding was blocked via a one-hour incubation with blocking reagent at room temperature (Bioworld Technology), and the samples were treated with $0.5 \%$ Triton X-100 in PBS before incubation with specific antibodies. The slides were incubated with a mouse monoclonal anti-MAP2 antibody (Abcam) at a 1:50 dilution overnight at $4^{\circ} \mathrm{C}$. After being washed with PBS, the slides were incubated with Flur ${ }^{\circledR}$ 488-Conjugated AffiniPure Goat AntiMouse $\operatorname{IgG}(\mathrm{H}+\mathrm{L})$ at a dilution of 1:50 for one hour at room temperature. After a second wash with PBS, the slides were incubated with DAPI (4,6-Diamidino-2-phenylindole dihydrochloride) at a 1:400 dilution for $5 \mathrm{~min}$ at room temperature. Finally, the slides were analyzed under a confocal laser scanning microscope.

\section{Real-time PCR}

Cortical neurons were plated on poly-L-lysinecoated $6 \mathrm{~cm}$ dishes at a density of $3 \times 10^{6}$ cells per dish. Total RNA was extracted from neurons at DIV 1, 4, 7, 11, 15 and 19 using TRIzol reagent (Ambion). Two micrograms of total RNA was reverse transcribed using the High Capacity cDNA 
Reverse Transcription Kit (AB Applied Biosystems), and $1 / 20$ of the RT products were used for real-time PCR amplification with the Power SYBR ${ }^{\circledR}$ Green PCR Master Mix (AB Applied Biosystems) in an Applied Biosystems 7500 Real-Time PCR system. The samples were analyzed in duplicate, and the experiments were performed with six biological replicates. The primers used in this assay were designed using the software Primer Express 3.0. The sequences of the primers are as follows: CDKL5-forward (5'AAAGGAGTGGCGACCTGAAA-3'), CDKL5reverse (5'-AGCAGCTTGCGAAGGGATTT-3'), $\mathrm{MeCP2}$ - forward GTCGCTCTGCTGGAAAGTATGA-3'), $\mathrm{MeCP2-}$ reverse CATTAGGGTCCAAGGAGGTGTCT-3’), Dnmt1-forward TAAAAAGCCAGCTATGCGACTTG-3'), Dnmt1-reverse $\left(5^{\prime}-\right.$ GCCAGGTAGCCTTCCTCAGA-3'), GAPDHforward (5'-GGGCTGGCATTGCTCTCA-3'), and $G A P D H$-reverse (5'CTGTAGCCATATTCATTGTCATACCA-3').

All reactions were normalized to $G A P D H$ and presented as relative expression changes in comparison to DIV 1 using the $\Delta \Delta \mathrm{CT}$ method $(\mathrm{n}=6)$.

\section{Antibodies}

The following primary antibodies were used in this study: rabbit polyclonal anti-CDKL5 (Abcam, ab191510), rabbit polyclonal anti-CDKL5 (Santa Cruz Biotechnology), mouse monoclonal antiMeCP2 (Abcam, ab50005), mouse monoclonal anti-MAP2 (Abcam), rabbit monoclonal antiDnmt1 (Cell Signaling Technology), and rabbit monoclonal anti- $\beta$-Actin (Cell Signaling Technology). The secondary antibodies used were the HRP Goat anti-Mouse IgG Antibody (Abgent) and the HRP Goat anti-Rabbit IgG Antibody (Abgent).

\section{Western blotting}

Neurons were cultured on poly-L-lysine-coated 10 $\mathrm{cm}$ dishes at a density of $6 \times 10^{6}$ cells per dish. Cells were harvested at DIV1, 4, 7, 11, 15 and 19 and lysed in the N-PER ${ }^{\mathrm{TM}}$ Neuronal Protein Extraction Reagent (Thermo) with $1 \times$ Halt $^{\mathrm{TM}}$ Protease Inhibitor Cocktail and $1 \times$ EDTA (Thermo), (for structural details, see http://www.piercenet.com/product/halt-proteaseinhibitor-cocktail). After centrifugation at
$10,000 \times \mathrm{g}$ at $4^{\circ} \mathrm{C}$ for $10 \mathrm{~min}$, the supernatant was collected. Protein concentrations were determined using the Bradford protein assay. The samples were resolved via SDS-PAGE under denaturing conditions and transferred to nitrocellulose membranes (Pall Corporation). Non-specific binding was blocked using 5\% nonfat dry milk in $1 \times$ TBS with $0.05 \%$ Tween 20 for one hour at room temperature. Primary antibody incubation was performed initially at room temperature for two hours and then at $4^{\circ} \mathrm{C}$ overnight. Subsequently, horseradish peroxidase-conjugated secondary antibodies (1:5000) were applied and incubated at room temperature for 1.5 hours. The antibodies against CDKL5 (Abcam), MeCP2, Dnmt 1 and $\beta$-actin were diluted 1:100, 1:1000, 1:250, 1:1000. Protein detection was performed using the SuperSignal ${ }^{\circledR}$ West Pico Chemiluminescent Substrate (Thermo), and the blots were exposed using KODAK 4000 MM PRO Image Station. Protein quantification for each sample was performed via densitometric analysis of the film using the AlphaEaseFC software. $\beta$ actin was used as a loading control for all western blot analyses. Each sample was represented by two technical replicates, and the analysis included five biological replicates for each DIV sample.

\section{Co-immunoprecipitation}

Cortical neurons were plated on poly-L-lysinecoated $10 \mathrm{~cm}$ dishes at a density of $6 \times 10^{6}$ cells per dish. Cells were harvested at DIV 4 and lysed using the N-PER ${ }^{\mathrm{TM}}$ Neuronal Protein Extraction Reagent (Thermo) with $1 \times$ Halt $^{\mathrm{TM}}$ Protease Inhibitor Cocktail and $1 \times$ EDTA (Thermo). After centrifugation at $10,000 \times \mathrm{g}$ at $4^{\circ} \mathrm{C}$ for $10 \mathrm{~min}$, the supernatant was incubated with $4 \mu \mathrm{g}$ of antiCDKL5 antibody (Santa Cruz Biotechnology) for 6 hours at $4^{\circ} \mathrm{C}$ with agitation. At the end of the incubation, rProtein A Sepharose ${ }^{\mathrm{TM}}$ Fast Flow beads (GE Healthcare Life Sciences) were added and incubated overnight at $4^{\circ} \mathrm{C}$ with agitation. The beads were washed 3 times with the N-PER ${ }^{\mathrm{TM}}$ Neuronal Protein Extraction Reagent and mixed with $20 \mu 1$ of $5 \times$ reduced SDS sample buffer. The samples were resolved via SDS-PAGE and immunoblotted with antibodies against $\mathrm{MeCP} 2$ and Dnmt1.

\section{Statistical analysis}

All values are represented as the mean \pm SEM. The data were analyzed using one -way ANOVA. Least Significant Difference post-hoc tests were 
performed to measure the significance of differences between each set of two groups. Statistical significance was defined as $P<0.05$.

\section{RESULTS}

\section{Determination of the purity of cultured cortical neurons}

As previously reported, two CDKL5 splicing isoforms, CDKL5a and CDKL5b, are present in the developing rat brain. CDKL5a is the neuronspecific isoform, which was identified by immunoblotting lysates of cultured cortical neurons, glial cells, and mixed neuron-glia culture with a commercial polyclonal antibody against CDKL5 (Chen et al. 2010). In this study, we aimed to elucidate the function of CDKL5 during the development of primary rat cortical neurons but not glial cells. To achieve this goal, we first determined the purity of the cultured neurons. The primary neurons were co-stained with the neuronspecific marker MAP2 and DAPI to determine whether the cell culture was neuron-dominant. The microscopic images in the bottom panel show that the cultures used in this study contained a very high percentage of neuronal cells (Figure 1).

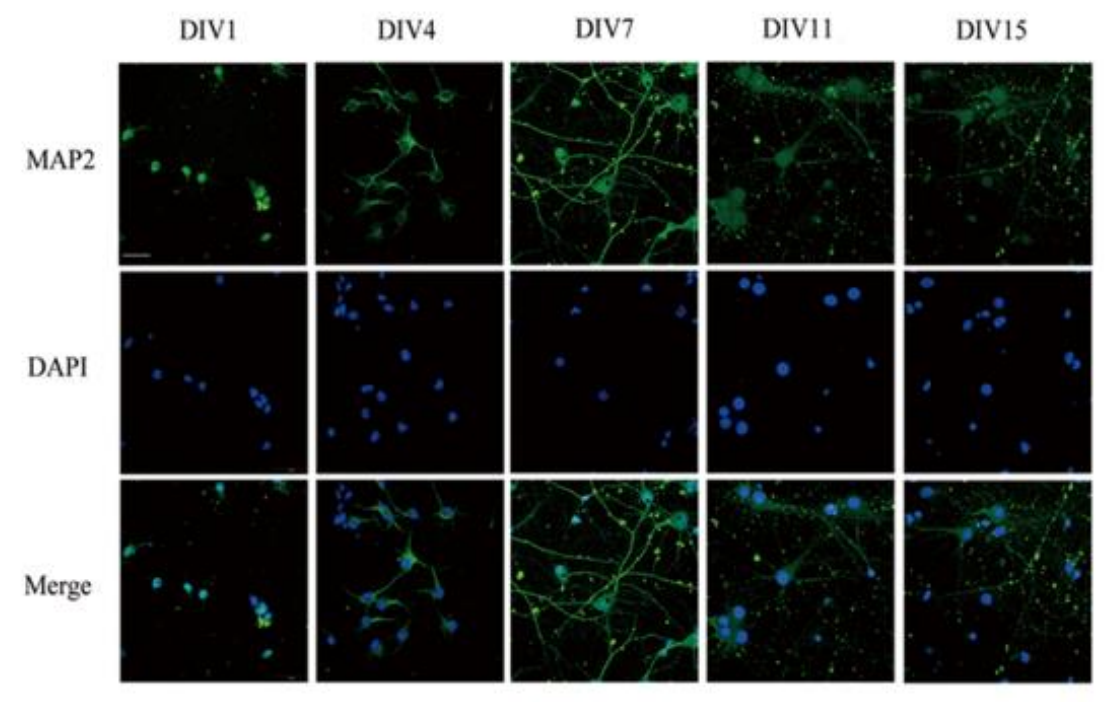

Fig. 1. Determination of the purity of primary rat cortical neuron cultures. Representative images for immunofluorescence staining of neurons at DIV 1, 4, 7, 11 and 15. Green, MAP2 (microtubule-associated protein 2), the neuron-specific marker. Blue, DAPI (4,6-Diamidino-2-phenylindole dihydrochloride) which stains DNA in the nuclei. Images were merged to check the purity of the cultures. For each slide, at leastfive images were captured from different fields. Scale bar, $30 \mu \mathrm{m}$.

CDKL5, MeCP2 and Dnmt1 have similar expression patterns at the transcriptional level in primary rat cortical neurons

We next examined the expression patterns of CDKL5, MeCP2 and Dnmt1 at the transcriptional level during the development of normal cultured cortical neurons. We chose the $\Delta \Delta \mathrm{CT}$ method of real-time PCR to explore the expression levels at DIV 4, 7, 11, 15 and 19 in comparison to DIV1 as a baseline value. The primers for CDKL5 were designed to only amplify cDNA of the CDKL5a splicing isoform, which is expressed primarily in neurons (Chen et al. 2010). The primers for $M e C P 2$ were designed to only amplify cDNA of $\mathrm{MeCP} 2 \mathrm{e} 1$, which is expressed primarily in the brain cortex (Cassel et al. 2006). Our results reveal that CDKL5 was highly expressed at DIV4 and DIV7, with a peak at DIV4. Expression began to decline gradually after DIV4 (Fig. 2A). MeCP2 expression reached its peak at DIV4 and then gradually decreased (Fig. 2B). The expression of Dnmt1 was high at DIV1 and then gradually decreased (Fig.2C). At DIV4, the expression levels of all three genes declined at approximately the same rate (Fig. 2D), suggesting that the three genes have similar expression patterns at the transcriptional level in cultured rat cortical neurons especially after DIV4. 
A

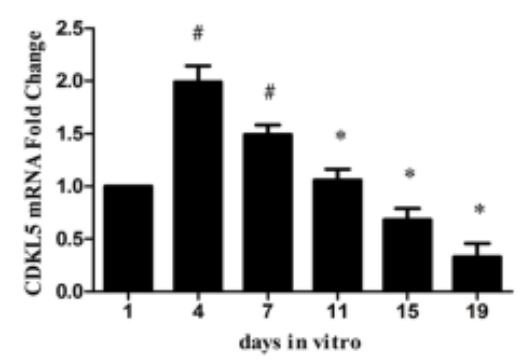

C

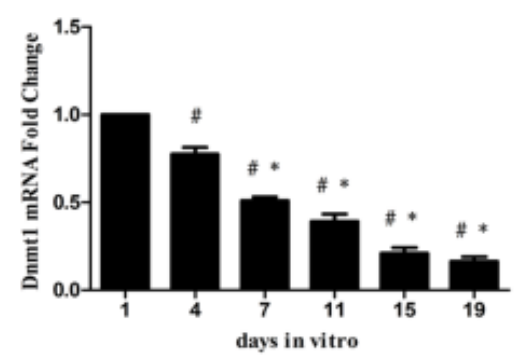

B

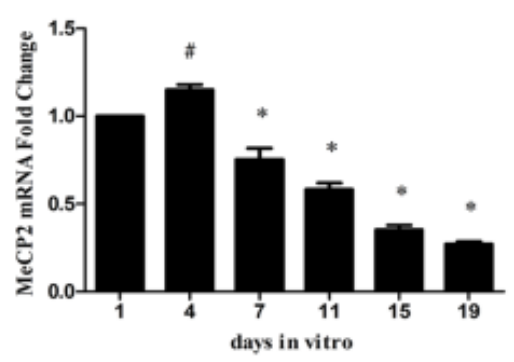

D

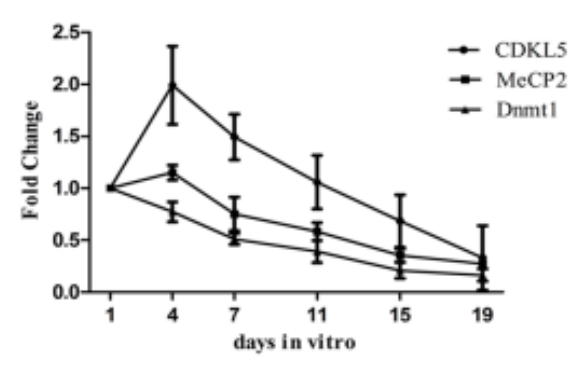

Fig. 2. The mRNA levels of CDKL5/MeCP2/Dnmt1 in cultured cortical neurons. The mRNA level was detectedby real-time PCR analysis. The mRNA levels were shown as values in comparison to that of DIV1. (A),Compared to baseline levels at DIV1, there is a significant 1.955 -fold increase in CDKL5 mRNA at DIV4, and1.4438-fold increase at DIV7. After DIV4, the CDKL5 mRNA levels decreased significantly. (B), MeCP2expression increased 1.109-fold at DIV4. After DIV4, the mRNA levels decreased significantly. (C), ForDnmt1, the mRNA levels significantly decreased right after DIV1 and DIV4. (D), The relationships of thesethree mRNA levels between DIV1 and DIV19. $n=6$, pound sign represents $\mathrm{P}<0.05$ (compared with DIV1), asterisk represents $\mathrm{P}<0.05$ (compared with DIV4).

CDKL5, MeCP2 and Dnmt1 have different expression patterns at the translational level in primary rat cortical neurons

Using western blotting, we detected the protein expression levels of the three molecules at the translational level. Our results revealed that CDKL5, MeCP2 and Dnmt1 exhibit different protein expression levels. The data show that the expression of the CDKL5 protein increased gradually, but not obviously reaching a peak at
DIV19 (Fig. 3A). MeCP2 expression increased in the cultured neurons, with a peak at DIV11 and a subsequent decrease (Fig. 3B). Compared with CDKL5, the observed increase in MeCP2 expression was more significant. The expression of Dnmt1 was slightly increased at DIV4, and protein expression gradually decreased at subsequent time points (Fig. 3C), which is inversely correlated to the pattern observed for CDKL5 after DIV4. 


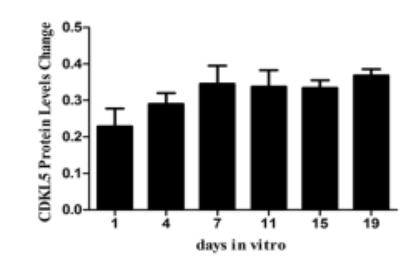

C

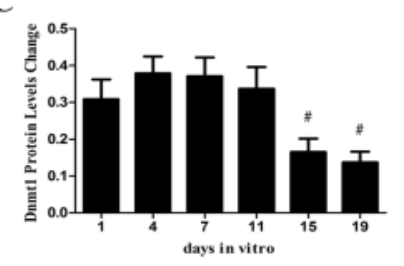

$\mathrm{E}$

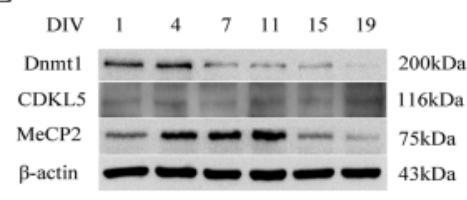

B

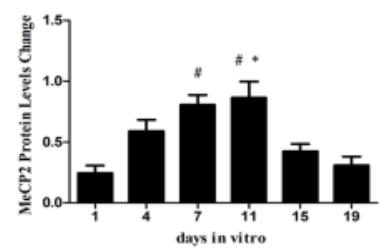

D

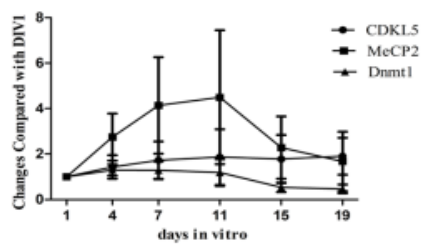

Fig. 3. Protein levels of CDKL5, MeCP2 and Dnmt1 in cultured cortical neurons. The expression levels ofproteins was detected by western blot analysis. (A) For CDKL5, the expression of the protein increasedgradually but not obviously reaching a peak at DIV19. (B) For MeCP2, there is a significant increase at DIV7and DIV11 in comparison to DIV1, then decreased at DIV15 and DIV19. (C) For Dnmt1, there is a slightlyincrease at the first few days before it significantly decreased at DIV15 and DIV19 in comparison to DIV1. (D) The relationships of these three protein levels between DIV1 and DIV19. (E) Representative images ofwestern blot analysis. $\beta$-actin is the internal control. $\mathrm{n}=5$, pound sign represents $\mathrm{P}<0.05$ (compared with DIV1), asterisk represents $\mathrm{P}<0.05$ (compared with DIV4).

\section{CDKL5 interacts with MeCP2 and Dnmt1 in primary rat cortical neurons}

To elucidate whether CDKL5 can bind to MeCP2 and Dnmt1 during rat neuronal in vitro differentiation, co-immunoprecipitation was performed. Incubation of polyclonal anti-CDKL5 with primary neuronal cell lysate samples at DIV4 and subsequent western blot analysis of $\mathrm{MeCP} 2$ and Dnmt1 revealed that an interaction occurs among endogenous CDKL5, MeCP2 and Dnmt1.

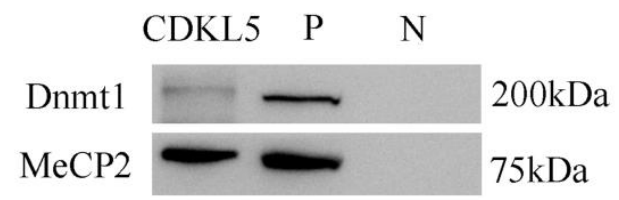

Fig. 4. Characterization of interaction among CDKL5, $\mathrm{MeCP} 2$ and Dnmt1 in cultured cortical neurons. Theprotein-protein interaction was detected using Coimmunoprecipitation. Cell lysates of cultured corticalneurons at DIV4 were immunoprecipitated with polyclonal anti-CDKL5. Eluates from the rProtein ASepharoseTM Fast Flow beads were analyzed using western blot to detect $\mathrm{MeCP} 2$ and Dnmt1. Input: positivecontrol, the whole cell extraction. $\mathrm{N}$ : negative control, the washing buffer collected when wash the beads thethird time. In the eluates from the beads ("CDKL5" line), both $\mathrm{MeCP} 2$ and Dnmt1 were detected.

\section{DISCUSSION}

CDKL5, which is a gene that has been mapped to chromosome Xp22, was first identified in 1998 using the positional cloning approach (Montini et al. 1998). Within the next few years, CDKL5 mutations had been found in patients with intellectual disability, infantile spasms, early onset seizures and encephalopathies (Archer et al. 2006; Evans et al. 2005; Kalscheuer et al. 2003; Nemos et al. 2009; Tao et al. 2004; Weaving et al. 2004). 
Importantly, mutations in CDKL5 cause the Hanefeld variant of Rett syndrome, in which the normal perinatal period is soon followed by the appearance of seizures with other symptoms, such as acquired microcephaly and stereotypic midline hand movements (Scala et al. 2005). Interest in the relationship between the proteins CDKL5 and $\mathrm{MeCP} 2$ has increased greatly in recent years, as mutations in these two genes cause similar clinical manifestations.

DNA methylation is an epigenetic regulation mechanism. Dnmt1 is an enzyme that recognizes and methylates hemimethylated $\mathrm{CpG}$ after DNA replication and maintains methylation patterns. Amino acids $650-850$ of CDKL5 have been reported to contain sequences that bind to Dnmt1 (1-290), and the kinase CDKL5 can phosphorylate Dnmt1 (1-290) in the presence of DNA (Kameshita et al. 2008). It is typically believed that methylated DNA will not be transcribed. $\mathrm{MeCP} 2$ is a protein that binds to methyl-DNA to repress gene expressions. A previous study demonstrated that Dnmt1 associates with MeCP2 to maintain DNA methylation in vivo (Kimura and Shiota 2003). Consistent with the ability of CDKL5 to phosphorylate both MeCP2 and Dnmt1, we hypothesize that these three proteins may interact with each other and function in the neuronal development process and synaptic plasticity. According to this hypothesis, CDKL5 binds both MeCP2 and Dnmt1 and phosphorylates those two proteins. After being phosphorylated, Dnmt1 can methylate hemimethylated $\mathrm{CpG}$ motifs following DNA replication, and MeCP2 can bind to methyl-DNA to repress the expression of target genes whose silence is important for the appropriate development of the nervous system. If mutations in CDKL5 destroy its kinase activity or its ability to bind to MeCP2 and Dnmt1, the functions of MeCP2 and Dnmt1 could be impaired. As a result, genes, that are supposed to be silenced, would be transcribed, and the resulting proteins could disturb normal nervous system development and cause clinical manifestations.

Our study aims to explore the expression patterns of CDKL5, MeCP2 and Dnmt1 in primary rat cortical neurons and ask whether these proteins interact with each other during the normal neuronal development process without the intervention of drugs or other conditions. The results show that at the transcriptional level, CDKL5, MeCP2 and Dnmt1 have similar expression patterns (Fig. 2D), but at the translational level, CDKL5 has a different expression profile than MeCP2 and Dnmt1 (Fig. 3D). Based on the western blotting data we found that while the expression of CDKL5 increased gradually, MeCP2 expression increased more significantly. However, the expression of Dnmt1 gradually decreased at the same time. Different mRNA and protein expression profiles may occur due to the regulation of proteins at the translational level.

Importantly, the results of our experiments are different from those of previous studies, especially at the mRNA level. Mari and his colleagues explored CDKL5 and MeCP2 mRNA expression profiles in embryonic and postnatal mouse brains. In situ hybridization results showed that CDKL5 expression is barely detectable during the late stages of embryogenesis in comparison to $\mathrm{MeCP} 2$ but strongly enhanced from P1 onwards. In the early postnatal days, a strong enhancement of CDKL5 expression was observed, with a stable peak at P10, which is similar to the pattern observed for MeCP2 (Mari et al. 2005). Jung, B.P. et al. found that $\mathrm{MeCP} 2 \mathrm{mRNA}$ expression in the developing rat cortex is continuously strong from E14 to adulthood (Jung et al. 2003). However, our data from cultured neurons show that mRNA expression of CDKL5 and MeCP2 increases at DIV4 and then decreases (Fig.2A and B). Importantly, we are the first to explore the mRNA expression of CDKL5, MeCP2 and Dnmt1 in primary rat cortical neurons, which provides a new approach to the delineation of the mRNA expression profiles of these three genes during neuronal differentiation.

At the protein level, our results show that CDKL5 expression is increased during neuronal development (Fig. 3A). This finding is consistent with two previous studies (Chen et al. 2010; Rusconi et al. 2011), which explored the expression patterns of CDKL5 in primary rat cortical neurons and primary mouse hippocampal neurons respectively. With respect to $\mathrm{MeCP} 2$, its expression increases from DIV1 to DIV11 before it undergoes a sharp decline (Fig. 3B). This pattern is to some extent similar that reported in the study of Mullaney, who found that MeCP2 protein levels increase from $\mathrm{P} 0$ to $\mathrm{P} 4$ in cortical plate cells of rats (Mullaney et al. 2004). Our data concerning the protein expression of Dnmt1 (Fig. 3C) are not consistent withthose reported in a study in which Dnmt1 protein levels were very 
stable during normal postnatal development of the mouse brain (Chestnut et al. 2011). The difference between these findings may be due to the use of different tissues or methods in the two studies.

Finally, we investigated whether any interaction occurs among CDKL5, MeCP2 and Dnmt1 during normal neuron development. Our results show that CDKL5 can bind to MeCP2 and Dnmt1 in cultured cortical neurons. In contrast to published works (Kameshita et al. 2008; Kimura and Shiota 2003; Mari et al. 2005) in which interactions were verified using GST pull-down assays or coimmunoprecipitation experiments with exogenous proteins transfected into cell lines, we carried out co-immunoprecipitation experiments on cell lysates of primary rat cortical neurons. We found that endogenous CDKL5 interact with MeCP2 and Dnmt1, suggesting that these three proteins might be functionally linked during neuronal development.

In summary, we demonstrated that the expression of CDKL5, MeCP2 and Dnmt1 are correlated at both the transcriptional and translational levels. CDKL5, interacts with MeCP2 and Dnmt1, suggesting that these proteins might be functionally linked in the nervous system. Future studies are needed to further investigate the relationships among CDKL5, MeCP2 and Dnmt1, and their individual functions in neural development and synaptic plasticity.

\section{ACKNOWLEDGEMENTS}

This study was supported by grants from the China National Natural Science Foundation (81070911). Thanks to Dingfang $\mathrm{Bu}$ for advice on real-time PCR and western blot and to Hansen Wang for advice on writing this paper.

\section{REFERENCES}

Archer HL, Evans J, Edwards S, Colley J, NewburyEcob R, O'Callaghan F, et al.: CDKL5 mutations cause infantile spasms, early onset seizures, and severe mental retardation in female patients. J Med Genet (2006) 43:729-734.

Cassel S, Carouge D, Gensburger C, Anglard P, Burgun $\mathrm{C}$, Dietrich JB, et al.: Fluoxetine and cocaine induce the epigenetic factors MeCP2 and MBD1 in adult rat brain. Mol Pharmacol (2006) 70:487492.
Chen Q, Zhu YC, Yu J, Miao S, Zheng J, Xu L, et al.: CDKL5, a protein associated with rett syndrome, regulates neuronal morphogenesis via Rac1 signaling. J Neurosci (2010) 30:12777-12786.

Chestnut BA, Chang Q, Price A, Lesuisse C, Wong M, Martin LJ: Epigenetic regulation of motor neuron cell death through DNA methylation. J Neurosci (2011) 31:16619-16636.

Evans JC, Archer HL, Colley JP, Ravn K, Nielsen JB, Kerr A, et al.: Early onset seizures and Rett-like features associated with mutations in CDKL5. Eur J Hum Genet (2005) 13:1113-1120.

Jung BP, Jugloff DG, Zhang G, Logan R, Brown S, Eubanks JH: The expression of methyl $\mathrm{CpG}$ binding factor $\mathrm{MeCP} 2$ correlates with cellular differentiation in the developing rat brain and in cultured cells. J Neurobiol (2003) 55:86-96.

Kalscheuer VM, Tao J, Donnelly A, Hollway G, Schwinger E, Kubart S, et al.: Disruption of the serine/threonine kinase 9 gene causes severe $\mathrm{X}$ linked infantile spasms and mental retardation. Am J Hum Genet (2003) 72:1401-1411.

Kameshita I, Sekiguchi M, Hamasaki D, Sugiyama Y, Hatano N, Suetake I, et al.: Cyclin-dependent kinase-like 5 binds and phosphorylates DNA methyltransferase 1. Biochem Biophys Res Commun (2008) 377:1162-1167.

Kimura H, Shiota K: Methyl-CpG-binding protein, $\mathrm{MeCP} 2$, is a target molecule for maintenance DNA methyltransferase, Dnmt1. J Biol Chem (2003) 278:4806-4812.

Lin C, Franco B, Rosner MR: CDKL5/Stk9 kinase inactivation is associated with neuronal developmental disorders. Hum Mol Genet (2005) 14:3775-3786.

Mari F, Azimonti S, Bertani I, Bolognese F, Colombo E, Caselli R, et al.: CDKL5 belongs to the same molecular pathway of $\mathrm{MeCP} 2$ and it is responsible for the early-onset seizure variant of Rett syndrome. Hum Mol Genet (2005) 14:1935-1946.

Montini E, Andolfi G, Caruso A, Buchner G, Walpole SM, Mariani M, et al.: Identification and characterization of a novel serine-threonine kinase gene from the Xp22 region. Genomics (1998) 51:427-433.

Mullaney BC, Johnston MV, Blue ME: Developmental expression of methyl- $\mathrm{CpG}$ binding protein 2 is dynamically regulated in the rodent brain. Neuroscience (2004) 123:939-949.

Nemos C, Lambert L, Giuliano F, Doray B, Roubertie A, Goldenberg A, et al.: Mutational spectrum of CDKL5 in early-onset encephalopathies: a study of a large collection of French patients and review of the literature. Clin Genet (2009) 76:357-371. 
Rusconi L, Kilstrup-Nielsen C, Landsberger N: Extrasynaptic N-methyl-D-aspartate (NMDA) receptor stimulation induces cytoplasmic translocation of the CDKL5 kinase and its proteasomal degradation. J Biol Chem (2011) 286:36550-36558.

Scala E, Ariani F, Mari F, Caselli R, Pescucci C, Longo I, et al.: CDKL5/STK9 is mutated in Rett syndrome variant with infantile spasms. J Med Genet (2005) 42:103-107.

Tao J, Van Esch H, Hagedorn-Greiwe M, Hoffmann K, Moser B, Raynaud M, et al.: Mutations in the Xlinked cyclin-dependent kinase-like 5 (CDKL5/STK9) gene are associated with severe neurodevelopmental retardation. Am J Hum Genet (2004) 75:1149-1154.
Weaving LS, Christodoulou J, Williamson SL, Friend KL, McKenzie OL, Archer H, et al.: Mutations of CDKL5 cause a severe neurodevelopmental disorder with infantile spasms and mental retardation. Am J Hum Genet (2004) 75:10791093.

Zhang Y, Wang H, Pan H, Bao X, Li M, Jin J, et al.: Gene delivery into primary cerebral cortical neurons by lentiviral vector. Cell Biol Int (2006) 30:777-783.

Received: September 09, 2015; Accepted: January 11,2016; 